Research Article

\title{
An Improved Strategy for Genetic Evolutionary Structural Optimization
}

\author{
Nannan Cui, ${ }^{1}$ Shiping Huang $\mathbb{D},^{2,3}$ and Xiaoyan Ding ${ }^{4}$ \\ ${ }^{1}$ School of Transportation Engineering, Shandong Jianzhu University, Jinan 250101, China \\ ${ }^{2}$ School of Civil Engineering and Transportation, South China University of Technology, Guangzhou 510640, China \\ ${ }^{3}$ Sino-Singapore International Joint Research Institute, Guangzhou, China \\ ${ }^{4}$ Shandong Hi-Speed Group Co., Ltd., Jinan 250098, China \\ Correspondence should be addressed to Shiping Huang; ctasihuang@scut.edu.cn
}

Received 7 January 2020; Revised 29 September 2020; Accepted 16 November 2020; Published 27 November 2020

Academic Editor: Flavio Stochino

Copyright $\odot 2020$ Nannan Cui et al. This is an open access article distributed under the Creative Commons Attribution License, which permits unrestricted use, distribution, and reproduction in any medium, provided the original work is properly cited.

\begin{abstract}
Genetic evolutionary structural optimization (GESO) method is an integration of the genetic algorithm (GA) and evolutionary structural optimization (ESO). It has proven to be more powerful in searching for global optimal response and requires less computational efforts than ESO or GA. However, GESO breaks down in the Zhou-Rozvany problem. Furthermore, GESO occasionally misses the optimum layout of a structure in the evolution for its characteristic of probabilistic deletion. This paper proposes an improved strategy that has been realized by MATLAB programming. A penalty gene is introduced into the GESO strategy and the performance index (PI) is monitored during the optimization process. Once the PI is less than the preset value which means that the calculation error of some element's sensitivity is too big or some important elements are mistakenly removed, the penalty gene becomes active to recover those elements and reduce their selection probability in the next iterations. It should be noted that this improvement strategy is different from "freezing," and the recovered elements could still be removed, if necessary. The improved GESO performs well in the Zhou-Rozvany problem. In other numerical examples, the results indicate that the improved GESO has inherited the computational efficiency of GESO and more importantly increased the optimizing capacity and stability.
\end{abstract}

\section{Introduction}

Topology optimization has played a significant role in machinery manufacturing and civil engineering and has rapidly developed since Prager and Rozvany [1] formulated the first general theory, termed "optimal layout theory." Topology optimization aims to obtain the optimum layout of materials from the initial design domain, which can efficiently increase material utilization. The homogenization theory of periodic media was first introduced by Bendsøe and Kikuchi [2] to solve the continuum topology optimization. Subsequently, two mainstream methods were proposed, solid isotropic material with penalization (SIMP) $[3,4]$ and evolutionary structural optimization (ESO) $[5,6]$. Though proposed late, topological derivative-based [7] and level-set methods [8, 9] also show great potential for further development. Recently, a method named Topology Optimization of Binary Structures
(TOBS) was developed by Sivapuram and Picelli [10] and Sivapuram et al. [11]. TOBS uses binary variables and works well for a wide range of problems investigated [12].

ESO is based on the simple idea that the initial design domain (structure) evolves towards optimization by systematically removing inefficient elements (material). BESO (bidictionary evolutionary structural optimization) $[13,14]$ is a derivate algorithm based on ESO which allows materials to be added and removed. BESO improves the robustness of the solution process compared to ESO. For its simple form and effectivity, ESO/BESO has been successfully used for actual engineering, including the Akutagawa office building in Japan [15] and Qatar International Exhibition Center [16]. Yunzhen $\mathrm{He}$ et al. [17] integrated BESO with genetic algorithms to create diverse and efficient structural designs. $\mathrm{ESO} / \mathrm{BESO}$ is recognized to be a powerful and promising technique and the application has been extended to the 
design of energy-absorbing structure [18, 19], multiple constraints' optimization problem [20], dynamic reliabilitybased topology optimization [21], concurrent topology optimization on both macro- and microscale [22], and numerous others. However, it has been criticized that the results from ESO are likely to be local optimums [23] and even the BESO cannot help. GESO (genetic evolutionary structural optimization) [24], an integration of genetic algorithm (GA) and ESO, proposed by Liu to cope with the local optimization problem of ESO, also failed under some circumstances.

For the failure of ESO, many researchers have attempted to improve it. These methods include the following: (1) Freezing the elements next to the supports [25]: in fact, in some structures, not all the supports are necessary. In situations where unnecessary elements are frozen, the uneconomical result will be obtained. (2) Refining mesh $[26,27]$ : attributing the failure of ESO to mesh sensitivity, some researchers have attempted to refine the mesh to get an optimal result. However, although mesh refinement can avoid some breakdowns, the user of ESO may never know which mesh density is the most suitable to get an optimal result. (3) Using rigorous resizing formulae [28]: secondorder sensitivities are suggested to prevent the failure. This method seems to be reasonable but its use in practice would increase significantly the computational effort. (4) Global difference method [29]: this improvement will search for "singular elements" during the evolution. The elements removed in one iteration whose sum of element strain energy is "much smaller" than that of the whole structure will be defined as "singular elements." The singular elements will be recovered and then the ESO will choose new elements to be removed by the global difference method. This improvement may have two shortcomings. Firstly, it is difficult to quantify "much smaller" as mentioned above to find those singular elements. Secondly, the global difference method is prohibitively expensive. (5) Soft-kill method [30, 31]: this improvement replaces elements to be removed with elements of low density. In doing so, the "wrongly removed" elements can be reserved. However, this method is entirely different from the concept of ESO and sacrifices the ESO's favorable characteristic of " $0-1$ discreteness."

Besides the imperfection in some situations, GESO provided a new idea of referring probability to refine the ESO. This research focuses on the improvement strategy based on GESO. This paper is organized as follows: Section 1 gives a brief review of topology optimization, especially on ESO and its derivate algorithms. Section 2 discusses the methodological rationality of ESO and the reason for solution failure. Section 3 describes the concept and shortcoming of GESO. Section 4 introduces the improved GESO (IGESO) and its basic procedure. Section 5 provides two typical examples to examine the performance of GESO. The last section presents some discussions and main conclusions.

\section{ESO and Zhou-Rozvany Counterexample}

2.1. ESO Method. ESO uses the "hard-kill" approach which introduces finite element changes in the optimization process on the basis of certain criteria. The basic concept is that the optimal topology can be produced by gradually removing an inefficient element from the design domain. This method was first proposed based on a fully stressed state, so the criterion of element removal was von Mises stress. Subsequently, removal criteria based on sensitivities with a more solid theoretical basis were utilized, including stiffness, weight, and frequency.

In ESO, the cycle of finite element analysis and element removal is repeated to produce the optimal structure. When to stop, the procedure should be carefully considered since there is no convergence criterion involved in the ESO. Preassigned removed volume ratio (RV) and rejection ration (RR) have been used as stop criteria in the literature. However, RV and RR are not universal for all cases. The performance index (PI) proposed by Liang and Xie (1999) is excellent in measuring the efficiency of topologies obtained by the ESO and is considered to be a more reasonable stop criterion.

2.2. Sensitivity. A certain kind of optimization will use the corresponding sensitivity. This section will describe the sensitivity of ESO by the example of "stiffness optimization."

In a structural analysis using FEM, the static behavior of a structure can be described by

$$
\mathbf{K u}=\mathbf{P} \text {, }
$$

where Kis the global stiffness matrix, $\mathbf{u}$ is the nodal displacement vector, and $\mathbf{P i s}$ the nodal load vector.

The strain energy of the structure is defined as

$$
C=\frac{1}{2} \mathbf{P}^{\mathrm{T}} \mathbf{u},
$$

where $C_{i}=(1 / 2) \mathbf{u}_{i}^{\mathrm{T}} \mathbf{K}_{i} \mathbf{u}_{i}$ is the element strain energy, where $\mathbf{K}_{i}$ and $\mathbf{u}_{i}$ are the stiffness matrix and displacement vector of the $i$ th element.

According to Chu et al. [32], if the ith element is removed from a structure comprising $n$ finite elements, the stiffness matrix will be changed by $\Delta \mathbf{K}=\mathbf{K}^{*}-\mathbf{K}=-\mathbf{K}_{i}$, where $\mathbf{K}^{*}$ is the stiffness matrix of the resulting structure after removal of the $i$ th element and $\mathbf{K}_{i}$ is the stiffness matrix of the $i$ th element. It is assumed that the element removal has no effect on the load vector P. By ignoring a higher-order term and on the basis of equation (1), the change of the displacement vector can be defined as

$$
\Delta \mathbf{u}=-\mathbf{K}^{-1} \Delta \mathbf{K u} \text {. }
$$

After the removal of the $i$ th element, we obtain the following equation from equations (2) and (3):

$$
\Delta C=\frac{1}{2} \mathbf{P}^{\mathrm{T}} \Delta \mathbf{u}=-\frac{1}{2} \mathbf{P}^{\mathrm{T}} \mathbf{K}^{-1} \Delta \mathbf{K} \mathbf{u}=\frac{1}{2} \mathbf{u}_{i}^{\mathrm{T}} \mathbf{K}_{i} \mathbf{u}_{i}
$$

where $\mathbf{u}_{i}$ is the displacement vector of the $i$ th element.

Then the sensitivity number is

$$
\alpha_{i}=\frac{1}{2} \mathbf{u}_{i}^{\mathrm{T}} \mathbf{K}_{i} \mathbf{u}_{i}, \quad(i=1, \ldots, n) .
$$


Equation (5) indicates the change of the strain energy of structure due to the removal of the $i$ th element. Hence, the removal of the elements with the lowest values of $\alpha_{i}$ is the most effective in minimizing the strain energy of a structure.

2.3. Performance Index (PI). For maximum stiffness topology design, the topology optimization problem is commonly stated as follows:

$$
\begin{aligned}
& \min . V=\sum_{j=1}^{n} v_{j}\left(t_{j}\right) \\
& \text { s.t. } C \leq C^{*} \\
& \quad 0 \leq t_{j} \leq t^{\max }, j=1, \ldots, n,
\end{aligned}
$$

where $V$ is the volume of the design domain. $v_{j}$ is the volume of the $j$ th element. $t_{j}$ is the thickness of the jth element. $t^{\max }$ is the maximum allowable value of each design variable. $C$ is the absolute value of the mean compliance of the structure. $C^{*}$ is the prescribed limit of $C$. $n$ denotes the total number of elements in the design domain.

However, it should be noted that the designer may not know how many types of compliance should be constrained to obtain the optimal structure and then a trial-and-error process cannot be avoided using equation (6). Fortunately, the ESO method may be not on the basis of equation (6), but on the PI. PI can characterize the efficiency of a certain topology.

For a linear plane stress continuum structure, the stiffness matrix is a linear function of the element's thickness. But in the ESO process, the element's thickness is not changeable. In order to get a structure meeting the mean compliance constraint in each iteration, we can uniformly scale the element's thickness with a factor of $C_{0} / C^{*}$. The scaled volume of the initial design domain can be expressed as

$$
V_{0}^{S}=\left(\frac{C_{0}}{C^{*}}\right) V_{0}
$$

where $V_{0}$ is the actual volume of the initial design domain. $C_{0}$ is the mean compliance of the initial design domain.

Similarly, the scaled volume of the $i$ th iteration can be expressed as

$$
V_{i}^{S}=\left(\frac{C_{i}}{C^{*}}\right) V_{i}
$$

where $V_{i}$ is the actual volume of structure in the $i$ th iteration. $C_{i}$ is the mean compliance of structure in the $i$ th iteration.

Then, the efficiency of the topology in the ith iteration can be measured by

$$
P I=\frac{V_{0}^{S}}{V_{i}^{S}}=\frac{C_{0} V_{0}}{C_{i} V_{i}} .
$$

The purpose of topology optimization is to find the most efficient layout of material (topology). The higher value of the PI means a better performance of a topology. So the topology optimization objective should be maximizing the PI or equivalently minimizing the product of strain energy and volume. It should be noted that the prescribed limit compliance $C^{*}$ is not in the expression of PI, which means that the topology optimization should not be subject to any constraints. Tanskanen [33] proposed the optimization problem of ESO as expressed as follows:

$$
\begin{aligned}
& \min . \quad f(\{t\})=C V \\
& \text { s.t. } \quad 0 \leq t_{j} \leq t^{\max }, \quad j=1, \ldots, n .
\end{aligned}
$$

Minimizing the volume of a structure with compliance constraint can be achieved by maximizing the PI in the optimization process. Scaling the thickness of an optimal topology obtained can satisfy the actual mean compliance constraint. So PI and minimum-volume design are not contradictory.

2.4. Zhou-Rozvany Counterexample. The topology optimization of the continuum is complicated for a large number of variables. The ESO method offers an accessible and efficient way to solve different kinds of topology optimization problems and the results are quite promising. However, ESO and its derivate algorithms have been questioned by Zhou and Rozvany [25] who have demonstrated a case where ESO could produce a totally nonoptimal solution. The ZhouRozvany counterexample is an academic example and shown in Figure 1(a), where Young's modulus is unity and Poisson's ratio is zero. The element thickness may only be unity or zero. The single load case consists of a horizontal load of intensity 2.0 and a vertical load of intensity 1.0. The model is discretized with 100 four-node quadrangle elements.

In the first iteration, ESO removes the element $a$ for the lowest strain energy sensitivity (1.99). The obvious reason for this is the fact that this element of the vertical tie receives the least diffusion from the horizontal stress in the beam. The compliance of renewed structure increases sharply to 4317 from 388.5 of the original structure. This large increase in compliance value is caused by the fact that the vertical load is now transmitted by flexural action which consequently proves that the ESO method produces a highly nonoptimal design (Figure 1(b)). In fact, neither element $b$ nor $c$ rejected would lead to this breakdown. Even BESO cannot help to rectify the highly nonoptimal solution because the highest strain energy sensitivity value occurs in the element $b$ (Figure 1(a)). BESO, therefore, would insert a new element in that location (dotted element in Figure 1(b)). Zhou and Rozvany also gave a conceivable optimal solution for a volume fraction of 40\%, shown in Figure 1(c), with a compliance value of 1121 .

\section{GESO}

The GESO method is an integration of the GA and the ESO method. GESO takes the advantage of GA's excellent behavior in searching for global optimums. For the GESO method, each element in finite element analysis is an individual and has its own fitness value according to the magnitude of its sensitivity number where all elements in an initial domain constitute a whole population in GA. After a 


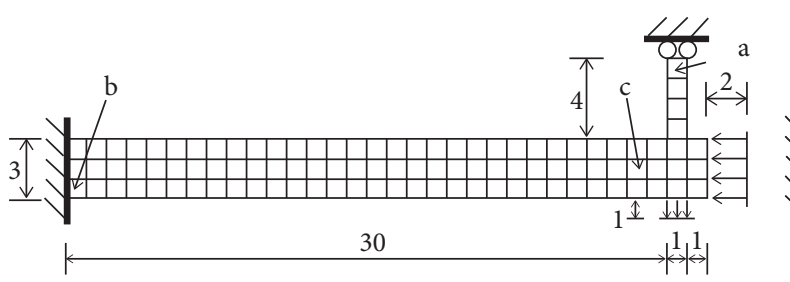

(a)

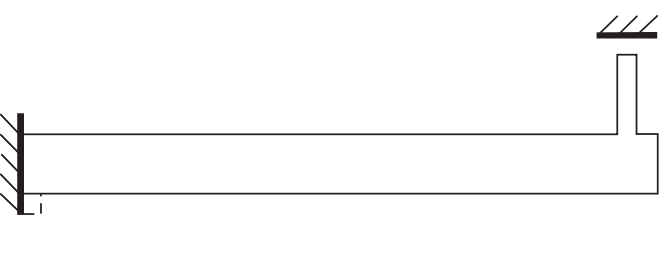

(b)

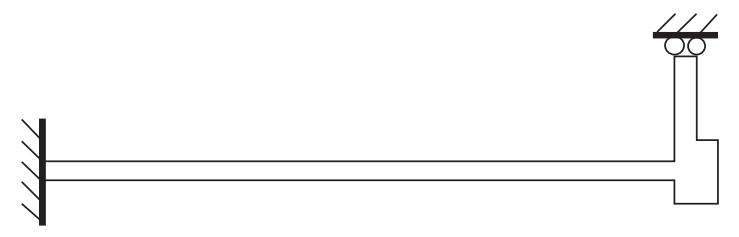

(c)

Figure 1: Zhou-Rozvany counterexample: (a) initial design domain with FE mesh, (b) topology given by ESO's rejection criterion for 1\% volume reduction, and (c) conceivable optimal solution for $60 \%$ volume reduction and the first line of the text.

number of generations, undeleted elements will converge to the optimal result of a global optimum than that of ESO.

GESO, to some extent, can avoid removing the element with sensitivity value calculated by error, but in some structures, GESO falls far short of the desired expectations, just like the Zhou-Rozvany counterexample. Theoretically, there are some possibilities for GESO to reserve the elements of the vertical tie and to catch the optimum result, but its chances are very small because the fitness of the vertical tie elements often stays at a relatively low level in the first evolutionary generations. The GESO proposer has realized this problem and improved it by introducing the interim thickness [31], which however is a "soft-kill" method and is entirely different from the concept of ESO. On the other hand, probabilistic removal of GESO, especially the introduction of crossover, would lead to accidental removal of some important elements, thereby causing the optimal structure to be missed.

\section{IGESO-Improved GESO}

4.1. Improvement Strategy. This paper puts forward a new improvement strategy taking advantages of the probability concept of GESO. The idea is achieved by monitoring the PI during the iteration. Once the PI is less than the preset value $\mathrm{PI}_{\text {th }}$ which means that the calculation error of some element's sensitivity is too big or some important elements are mistakenly removed, the penalty gene becomes active to recover those elements and reduce their selection probability in the next iterations. The new strategy is different from the "freezing method," and the punished elements still have the chance to be removed in the subsequent iterations. It should be noted that this improvement is based on the probabilistic removal of GESO and will not work in the certainty removal method.

We named the improved GESO as IGESO. In IGESO, the means to lower the removal probability of the "wrong elements" is as follows: before the optimization procedure, every element is arranged with a chromosome consisting of two parts: one part is $n$-bit length binary string made of number "1" (named as a status gene), while the other is a decimal number with the initial settling of value " 0 " (named as penalty gene). When the PI is less than $\mathrm{PI}_{\text {th }}$ in a certain iteration, the last removal elements will be recovered, and the penalty gene of these elements will be added 1 to reduce the removal probability, described as

$$
p_{i}^{\prime}=d^{c} p_{i}
$$

where $p_{i}^{\prime}$ is the reduced removal probability and $d \in(0,1)$ is the penalty coefficient whose value should be determined according to the requirement; however, 0.01 is a recommended value. $p_{i}$ is the removal probability calculated according to GESO [24].

The value of $\mathrm{PI}_{\text {th }}$ represents the requirement for the element's sensitivity calculating precision. When $\mathrm{PI}_{\text {th }}$ is set high, the requirement is high and the computational efficiency is also reduced. When $\mathrm{PI}_{\text {th }}$ is set low, even close to zero, IGESO is almost equal to GESO, which eventually describes the tolerance of all the computational errors of element's sensitivity. The author has researched a large number of topology optimization examples and considered that a value around 0.9 is reasonable. In fact, PI will drop sharply to a very small value when the important elements are mistakenly removed. For instance, in the Zhou-Rozvany counterexample, the PI drops to 0.089 when the element $a$ is removed.

\subsection{Basic Procedure of IGESO}

(a) Build up an original finite element model and determine boundary and load conditions.

(b) Impose an $n$-bit length binary string (status genes) and a decimal number (penalty gene) for chromosome. The values of status genes are all " 1 " to each element and the penalty gene is $0 . n$ is selected arbitrarily.

(c) Solve the structural static equation (6).

(d) Calculate the sensitivity of the elements and smooth the calculated value. 


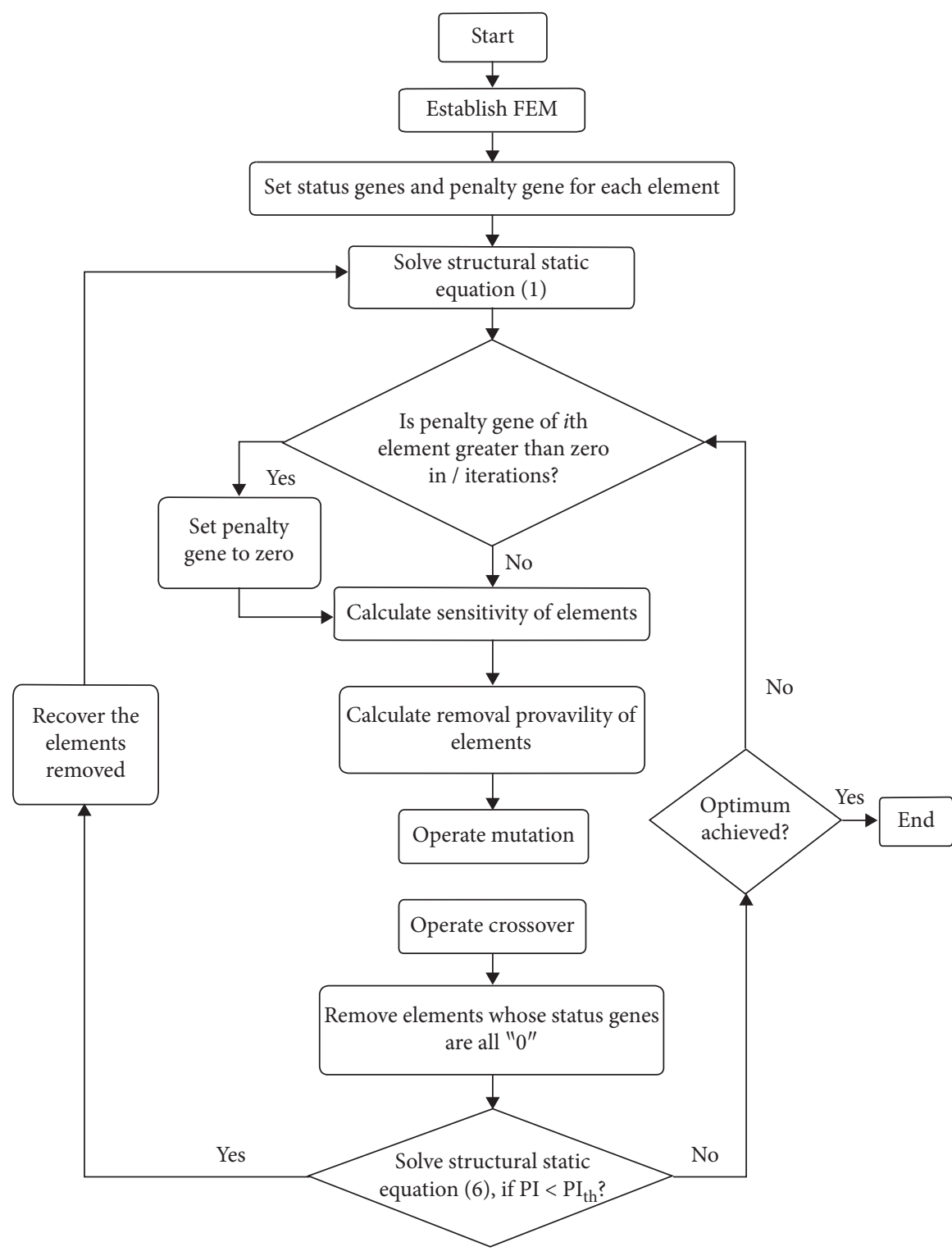

Figure 2: Flowchart of IGESO.

(e) All elements that stayed in the structure constitute the whole population. Rank individuals according to the sensitivity value and calculate the removal probability according to equation (11). Operate mutation over the last $m$ individuals according to the ranking. Here, the term mutation is slightly different than that of GA, which is only to change a nonzero status gene to zero.

(f) Operate crossover on status genes in population.

(g) Remove the elements whose status genes are all "0."

(h) Solve the structural static equation (6). If $\mathrm{PI}<\mathrm{PI}_{\text {th }}$ in this iteration, then recover the elements removed in step (g), add 1 to the penalty gene of those elements, and go to step (c). If $\mathrm{PI} \geq \mathrm{PI}_{\mathrm{th}}$ in this iteration, then go to step (d). (i) If the penalty gene of an element is greater than zero and has no changes in $l$ iterations, then change it to zero.

(j) Repeat steps (c) to (i) until an optimum result is reached or one of the constraints reaches its limit.

The flowchart of IGESO is shown in Figure 2. The improved strategy proposed has been realized by MATLAB programming in which the FEM software ANSYS is called in a loop to accomplish the FEM calculation.

\section{Examples}

5.1. Zhou-Rozvany Counterexample. The basic information has been described in Section 2. The optimization process of this example is somewhat special because the value of $P I$ will 


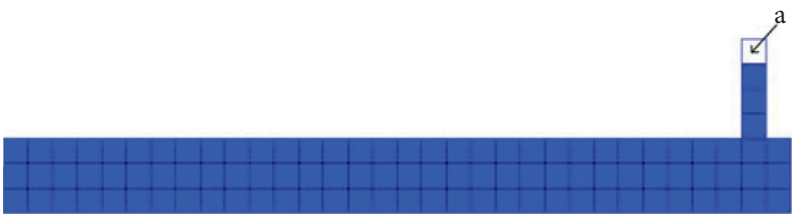

(a)

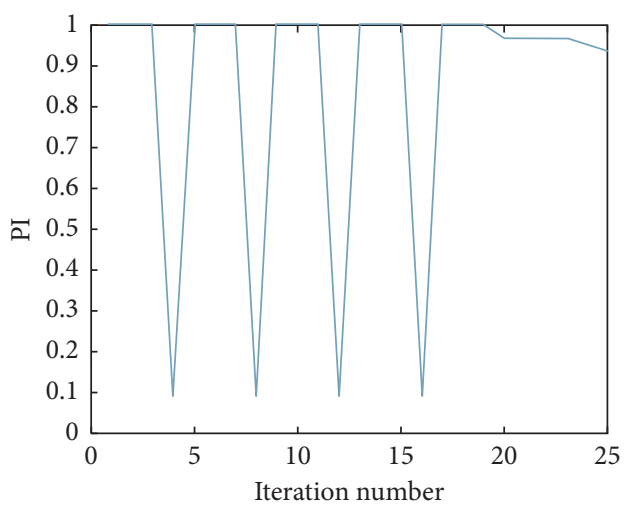

(c)

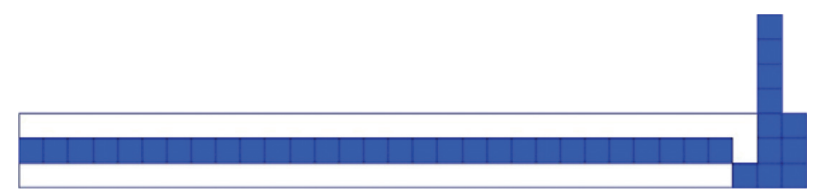

(e)

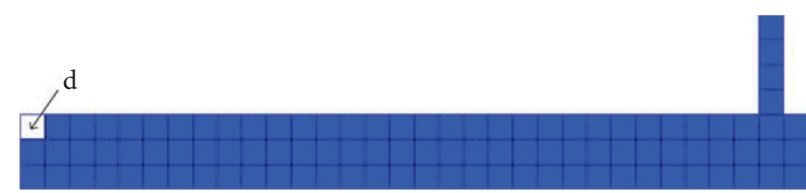

(b)

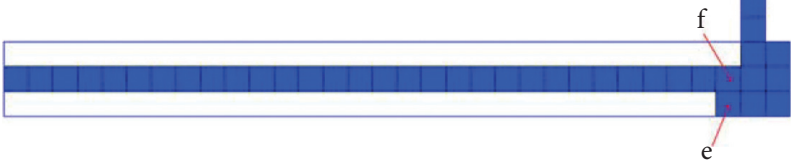

(d)

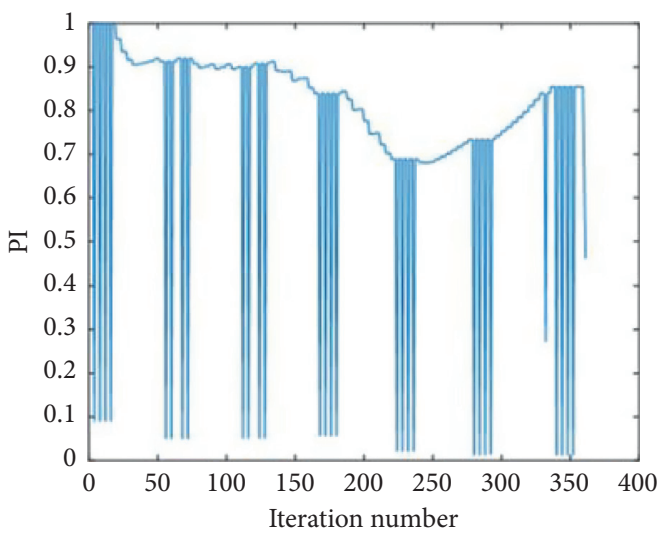

(f)

FIgURe 3: IGESO optimal design on Zhou-Rozvany counterexample: (a) topology after 4 iterations, (b) topology after 20 iterations, (c) PI curve of 24 iterations, (d) topology for 59\% volume reduction, (e) topology for $60 \%$ volume reduction, and (f) PI curve of the whole process.

decline with the removal of any element and never be greater than 1. Referring to the optimum solution given by Zhou and Rozvany [25], we set $\mathrm{PI}_{\text {th }}=0.347$. The other parameters were set according to GESO [24], $n=4, q=0.5, P_{c}=0.2$, and $P_{m}=1$.

As shown in Figure 3(a), in the fourth iteration (element removal for the first time) just like GESO and ESO, element $a$ is mistakenly removed. At the same time, it has been found that the value of PI drops sharply from 1 to 0.1 (Figure 3(c)). Then, the penalty gene is activated to recover the element $a$ and to reduce its removal probability in the next iteration. As a result, the value of PI augmented sharply. Such kind of sharp fluctuation in the PI curve illustrates that the penalty genes of certain elements are activated once. Because all those four elements of vertical tie receive little diffusion from the horizontal stress, they all have a large probability to remove until all of them are punished. After that, element $b$ is chosen to be removed, and PI is satisfied in this iteration (Figure 3(b)). After 59 elements were removed, an optimum structure shown in Figure 3(d) was achieved. The removal of element $e$ in the next iteration would bring the optimal solution just like Zhou and Rozvany predicted (Figure 3(c)). However, the IGESO solves this example by removing element $f$, and the strain energy of the total structure is $\mathbf{9 7 2}$, which is lower than 1121 predicted by Zhou and Rozvany. Correspondingly, the PI of the optimum structure is 0.4 , which is higher than 0.347 (Zhou and Rozvany's prediction).

By IGESO, the breakdown of the Zhou-Rozvany counterexample is avoided and more importantly, IGESO can get a better solution.

5.2. Simply Supported Beam under Three Concentrated Loads. The structure to be optimized is shown in Figure 4. Young's modulus is $207 \mathrm{GPa}$ and Poisson's ratio is 0.3 . The initial 


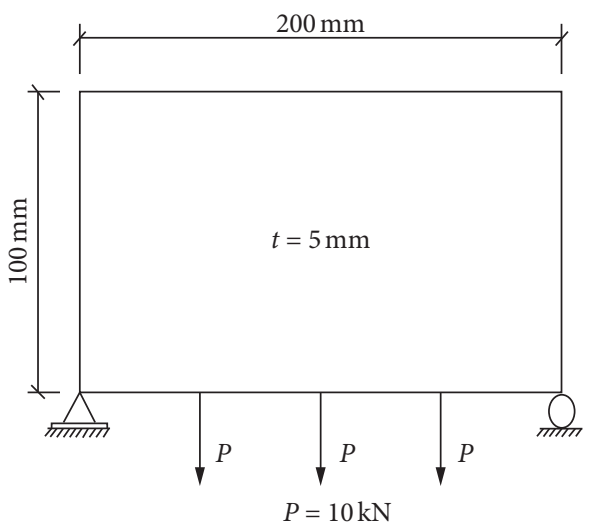

FIGURE 4: Simply supported beam under three concentrated loads.

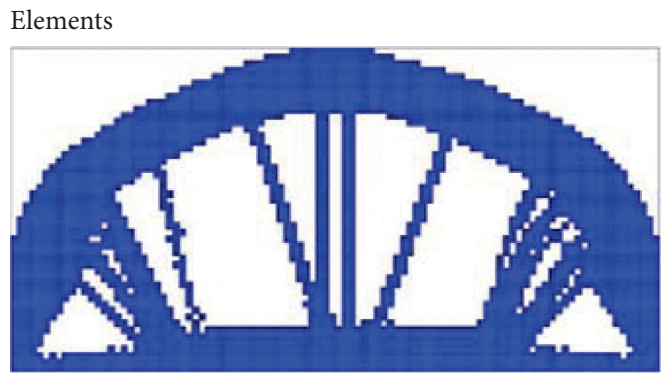

(a)

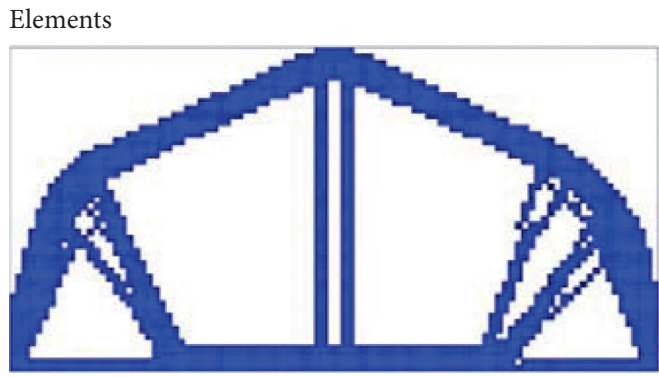

(c)

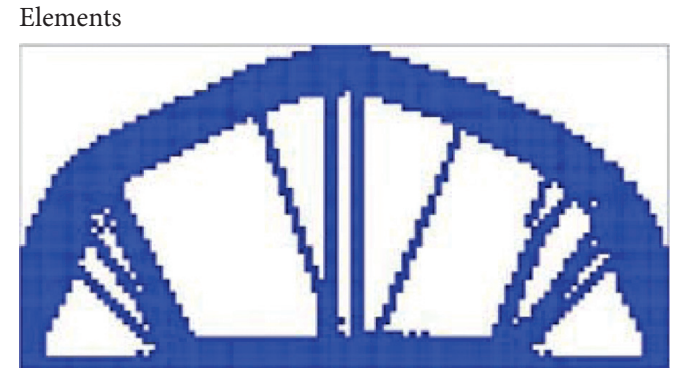

(b)

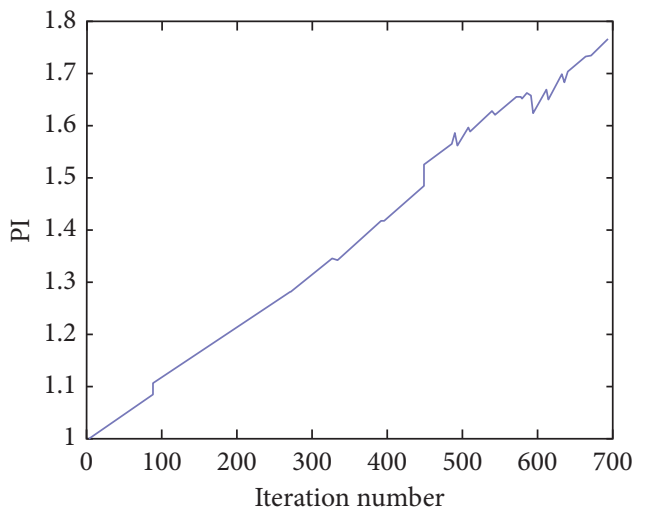

(d)

FIGURE 5: IGESO optimal design for simply supported beam: (a) topology for 50\% volume reduction, (b) topology for $60 \%$ volume reduction, (c) topology for $70 \%$ volume reduction, and (d) PI curve of the whole process.

design domain meshes with $2 \mathrm{~mm} \times 2 \mathrm{~mm}$ quadrangle elements. $\mathrm{PI}_{\mathrm{th}}=1$ is set to ensure that the topology optimum is better than the initial. Other parameters are set according to the literature [24]. In order to compare conveniently with the literature $[24,34], C_{\text {obj }}$ (reciprocal of PI) is used to measure the optimization results.

The optimal design from IGESO is given in Figure 5, and $C_{\text {obj }}$ by four algorithms is compared in Table $1 . C_{\text {obj }}$ by IGESO is closed to GESO and less than ESO and BESO, which indicates that both IGESO and GESO have powerful capacity in searching for better results. In fact, there is no fluctuation which means that the penalty genes activated are found in the PI curve (Figure 5(d)). Therefore, if not
TABLE 1: Comparison of the results from the four algorithms.

\begin{tabular}{lcccc}
\hline RV (\%) & IGESO & GESO [24] & ESO [24] & BESO [24] \\
\hline 50 & 0.636 & 0.626 & 0.665 & 0.637 \\
60 & 0.602 & 0.575 & 0.623 & 0.636 \\
70 & 0.564 & 0.532 & 0.579 & 0.584 \\
\hline
\end{tabular}

necessary, the IGESO will not decline the advantage of the computational cost of GESO.

The topology for $85 \%$ volume reduction by IGESO and GESO is given in Figure 6. It is obvious that GESO goes to nonoptimal results for some mistaken element removal 


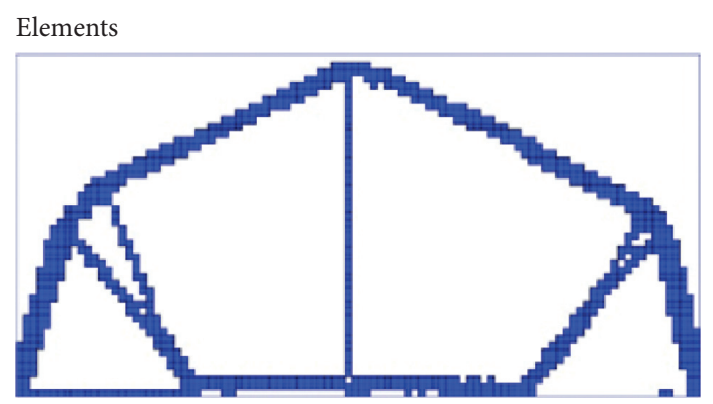

(a)

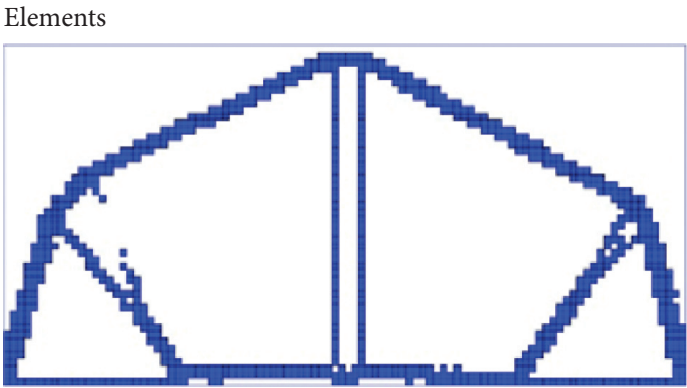

(c)

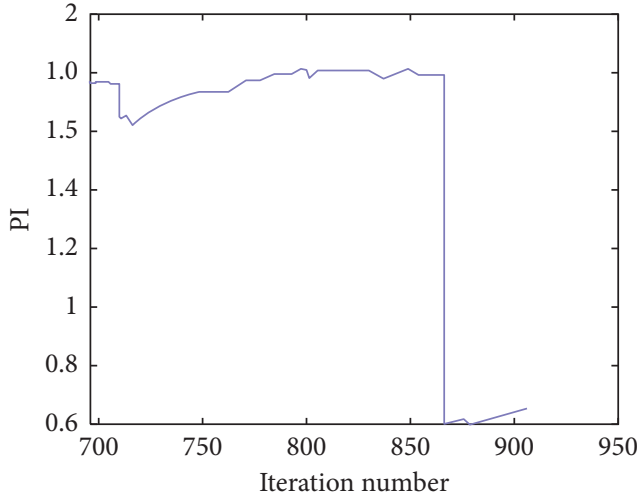

(b)

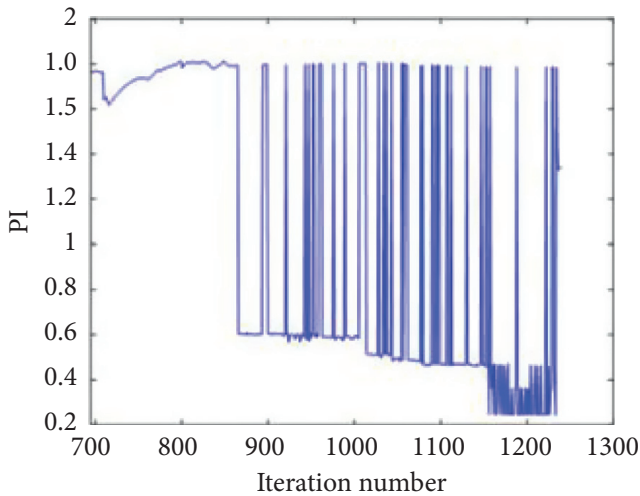

(d)

FIGURE 6: Comparison of the topology for $85 \%$ volume reduction by IGESO and GESO: (a) topology by GESO, (b) PI curve of GESO after 700 iterations, (c) topology by IGESO, and (d) PI curve of IGESO after 700 iterations.

(the horizontal bar on the lower right is destroyed as shown in Figure 6(a)). At the same time, the PI drops sharply to 0.6 (Figure $6(\mathrm{~b})$ ). Those mistakenly removed elements cannot be recovered by GESO in the later iterations, eventually causing the finally obtained results to be nonoptimum. However, IGESO can avoid the mistaken element removal which has been shown in Figure 6(c). There are numerous fluctuations in Figure 6(d), which means that the procedure is not very easy; however, an optimal solution for $85 \%$ volume reduction is presented finally whose PI is 1.34 .

\section{Conclusions}

An improvement strategy for GESO has been presented in this paper. Numerical examples considered herein have shown that the IGESO has inherited the computational efficiency of GESO, and more importantly, it has increased the optimization capacity and stability.

It is noteworthy to describe that, as a derivate algorithm of ESO, the IGESO takes the advantages of the probability concept of GESO and this improvement strategy has no effect on any certainty algorithm. The solution provided by GESO or IGESO may be different after each run of the program and the results presented here are the best ones obtained from several runs.

\section{Data Availability}

The data used to support the findings of this study are included within the article.

\section{Conflicts of Interest}

The authors declare that they have no conflicts of interest.

\section{Authors' Contributions}

Cui Nannan and Huang Shiping conceived the presented idea. Cui Nannan, Huang Shiping, and Ding Xiaoyan wrote the paper.

\section{Acknowledgments}

This paper was supported by Natural Science Foundation of China (NSFC) (Grants nos. 11672108 and 11202080) and Science and Technology Project of Shandong Transportation Department (2020B69). This work was also supported by "the Fundamental Research Funds for the Central Universities”. 


\section{References}

[1] W. Prager and G. I. Rozvany, "Optimization of structural geometry," in Dynamical Systems, pp. 265-293, Elsevier, Amsterdam, Netherlands, 1977.

[2] M. P. Bendsøe and N. Kikuchi, "Generating optimal topologies in structural design using a homogenization method," Computer Methods in Applied Mechanics and Engineering, vol. 71, pp. 197-224, 1988.

[3] G. I. N. Rozvany, M. Zhou, and T. Birker, "Generalized shape optimization without homogenization," Structural Optimization, vol. 4, no. 3-4, pp. 250-252, 1992.

[4] M. Zhou and G. I. N. Rozvany, "The COC algorithm, part II: topological, geometrical and generalized shape optimization," Computer Methods in Applied Mechanics and Engineering, vol. 89, no. 1-3, pp. 309-336, 1991.

[5] Y. M. Xie and G. P. Steven, "Shape and layout optimization via an evolutionary procedure," in Proceedings of the International Conference on Computational Engineering Science, Kobe, Japan, April 1992.

[6] Y. M. Xie and G. P. Steven, "A simple evolutionary procedure for structural optimization," Computers \& Structures, vol. 49, no. 5, pp. 885-896, 1993.

[7] J. Sokolowski and A. Zochowski, "Topological derivative in shape optimization," Encyclopedia of Optimization, pp. 3908-3918, Springer, Berlin, Germany, 2009.

[8] J. A. Sethian and A. Wiegmann, "Structural boundary design via level set and immersed interface methods," Journal of Computational Physics, vol. 163, no. 2, pp. 489-528, 2000.

[9] M. Y. Wang, X. Wang, and D. Guo, "A level set method for structural topology optimization," Computer Methods in Applied Mechanics and Engineering, vol. 192, no. 1-2, pp. 227-246, 2003.

[10] R. Sivapuram and R. Picelli, "Topology optimization of binary structures using Integer Linear Programming," Finite Elements in Analysis and Design, vol. 139, pp. 49-61, 2018.

[11] R. Sivapuram, R. Picelli, and Y. M. Xie, "Topology optimization of binary microstructures involving various non-volume constraints," Computational Materials Science, vol. 154, pp. 405-425, 2018.

[12] R. Picelli and R. Sivapuram, "Solving topology optimization with $\{0,1\}$ design variables and mathematical programming: the TOBS method," in Proceedings of the World Congress of Structural and Multidisciplinary Optimization, Beijing, China, May 2019.

[13] O. M. Querin, G. P. Steven, and Y. M. Xie, "Evolutionary structural optimisation (ESO) using a bidirectional algorithm," Engineering Computations, vol. 15, no. 8, pp. 1031-1048, 1998.

[14] X. Y. Yang, Y. M. Xie, G. P. Steven, and O. M. Querin, "Bidirectional evolutionary method for stiffness optimization," AIAA Journal, vol. 37, no. 11, pp. 1483-1488, 1999.

[15] H. Ohmori, H. Futai, T. Iijima, A. Muto, and Y. Hasegawa, "Structural design of office building by computational morphogenesis (structures)," AIJ Journal of Technology and Design, vol. 10, no. 20, pp. 77-82, 2004.

[16] C. Cui, H. Ohmori, and M. Sasaki, "Computational morphogenesis of 3D structures by extended ESO method," Journal of the International Association for Shell and Spatial Structures, vol. 44, pp. 51-61, 2003.

[17] Y. He, K. Cai, Z.-L. Zhao, and Y. M. Xie, "Stochastic approaches to generating diverse and competitive structural designs in topology optimization," Finite Elements in Analysis and Design, vol. 173, Article ID 103399, 2020.
[18] X. Huang, Y. M. Xie, and G. Lu, "Topology optimization of energy-absorbing structures," International Journal of Crashworthiness, vol. 12, no. 6, pp. 663-675, 2007.

[19] L. Li, G. Zhang, and K. Khandelwal, "Topology optimization of energy absorbing structures with maximum damage constraint," International Journal for Numerical Methods in Engineering, vol. 112, no. 7, pp. 737-775, 2017.

[20] Z. H. Zuo, Y. M. Xie, and X. Huang, "Evolutionary topology optimization of structures with multiple displacement and frequency constraints," Advances in Structural Engineering, vol. 15, no. 2, pp. 359-372, 2012.

[21] B. Xu, L. Zhao, W. Li, J. He, and Y. M. Xie, "Dynamic response reliability based topological optimization of continuum structures involving multi-phase materials," Composite Structures, vol. 149, pp. 134-144, 2016.

[22] W. M. Vicente, Z. H. Zuo, R. Pavanello, T. K. L. Calixto, R. Picelli, and Y. M. Xie, "Concurrent topology optimization for minimizing frequency responses of two-level hierarchical structures," Computer Methods in Applied Mechanics and Engineering, vol. 301, pp. 116-136, 2016.

[23] G. I. N. Rozvany, "A critical review of established methods of structural topology optimization," Structural and Multidisciplinary Optimization, vol. 37, no. 3, pp. 217-237, 2009.

[24] X. Liu, W.-J. Yi, Q. S. Li, and P.-S. Shen, "Genetic evolutionary structural optimization," Journal of Constructional Steel Research, vol. 64, no. 3, pp. 305-311, 2008.

[25] M. Zhou and G. I. N. Rozvany, "On the validity of ESO type methods in topology optimization," Structural and Multidisciplinary Optimization, vol. 21, no. 1, pp. 80-83, 2001.

[26] C. S. Edwards, H. A. Kim, and C. J. Budd, "An evaluative study on ESO and SIMP for optimising a cantilever tie-beam," Structural and Multidisciplinary Optimization, vol. 34, no. 5, pp. 403-414, 2007.

[27] X. Huang and Y. Xie, "A new look at ESO and BESO optimization methods," Structural and Multidisciplinary Optimization, vol. 35, pp. 89-92, 2008.

[28] G. I. N. Rozvany, "Stress ratio and compliance based methods in topology optimization-a critical review," Structural and Multidisciplinary Optimization, vol. 21, no. 2, pp. 109-119, 2001.

[29] H. Dan and L. Shutian, "The causative agent of invalidation and improvement strategy for evolutionary structural optimization," Chinese Journal of Computational Mechanics, vol. 31, pp. 310-314, 2014.

[30] G. I. N. Rozvany, O. M. Querin, Z. Gaspar, and V. Pomezanski, "Extended optimality in topology design," Structural and Multidisciplinary Optimization, vol. 24, no. 3, p. 257, 2002.

[31] X. Liu, The Research of Genitic Evolutionary Algorithm on Structrual Optimization, Hunan University, ; Changsha, China, 2007.

[32] D. N. Chu, Y. M. Xie, A. Hira, and G. P. Steven, "Evolutionary structural optimization for problems with stiffness constraints," Finite Elements in Analysis and Design, vol. 21, no. 4, pp. 239-251, 1996.

[33] P. Tanskanen, "The evolutionary structural optimization method: theoretical aspects," Computer Methods in Applied Mechanics and Engineering, vol. 191, no. 47-48, pp. 54855498, 2002.

[34] X. Yang, Y. Xie, G. Steven, and O. Querin, "Bi-directional evolutionary method for stiffness optimisation," in Proceedings of the 7th AIAA/USAF/NASA/ISSMO Symposium on Multidisciplinary Analysis and Optimization, St. Louis, MO, USA, September 1998. 